\title{
Using the Initial Systolic Time Interval to assess cardiac autonomic nervous function in Parkinson's disease
}

\author{
Jan H. Meijer ${ }^{1,3}$, Eskeline Elbertse ${ }^{2}$, Sanne Boesveldt ${ }^{2}$, Henk W. Berendse ${ }^{2}$ and Rudolph M. Verdaasdonk ${ }^{1}$ \\ 1. Department of Physics and Medical Technology, VU University Medical Center, Amsterdam, the Netherlands \\ 2. Department of Neurology, VU University Medical Center, Amsterdam, the Netherlands \\ 3.E-mail any correspondence to: jh.meijer@vumc.com
}

\begin{abstract}
The Initial Systolic Time Interval (ISTI) has been defined as the time difference between the peak electrical and peak mechanical activity of the heart. ISTI is obtained from the electro-cardiogram and the impedance cardiogram. The response of ISTI while breathing at rest and to a deep breathing stimulus was studied in a group of patients suffering from Parkinson's disease (PD) and a group of healthy control subjects. ISTI showed substantial variability during these manoeuvres. The tests showed that the variability of RR and ISTI was substantially different between PD patients and controls. It is hypothesized that in PD patients the sympathetic nervous system compensates for the loss of regulatory control function of the blood-pressure by the parasympathetic system. It is concluded that ISTI is a practical, additional and independent parameter that can be used to assist other tests in evaluating autonomic nervous control of the heart in PD patients.
\end{abstract}

Keywords: Initial systolic time interval, Impedance cardiography, Parkinson's disease, cardiac autonomic nervous function

\section{Introduction}

When the electrical impedance of a human thorax is measured a variation synchronous with the heart activity can be obtained. The time derivative of this variation is called the Impedance CardioGram (ICG). Numerous studies during the last decades have demonstrated that the origin of this ICG-signal is too complicated to make an interpretation of the amplitude as a simple one-way translation of cardiac stroke volume [1, 2, 3, 4]. However, Meijer et al [5] have pointed out that an interesting aspect of the ICG-signal can be found in the time relationships of this signal with the Electro CardioGram (ECG). Regardless of the multiple sources of the signal, the ICG reflects the mechanical aspects of cardiac activity [6], while the ECG reflects the electrical aspects. Therefore, by recording both signals simultaneously, the time difference between electrical and mechanical events in the cardiac cycle can be studied. In a previous report the Initial Systolic Time Interval (ISTI) was defined as the time difference between the peak electrical and peak mechanical activity of the heart [5], marked by the R-point in the ECG and by the C-point in the ICG. ISTI can be studied in various physiological conditions and diseases and may be helpful to evaluate pathological processes and rehabilitation processes. The use of ISTI for diagnostic purposes is attractive: both ECG and ICG signals can be obtained fast and easily. The measurements are noninvasive, of low burden to patients and are not restricted to a clinical environment. The required technical equipment is relatively inexpensive, portable and easy to operate.

Up to date little is known about the behaviour of ISTI in various physiological and clinical circumstances. There is a need for observational research to reveal the behaviour of the ISTI under these circumstances. This behaviour will also indicate the value of ISTI as an independent addition to cardiovascular reflex tests designed to diagnose autonomic nervous dysfunction [7]. The aim of the present study was to investigate the respiratory variation in ISTI (and RRinterval) at rest and during stimulated deep breathing in a group of patients suffering from Parkinson's disease (PD) and to compare these variations with a group of healthy control subjects.

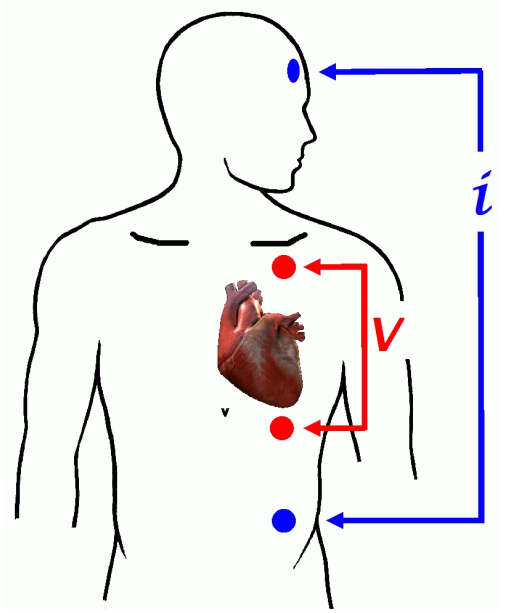

Fig.1: Electrode configuration for the measurement of ISTI: A small electrical current $\boldsymbol{i}$ was applied to the upper part of the body by means of the two outer electrodes. The two inner electrodes measured the subsequent electrical voltage difference $\boldsymbol{V}$ over the heart from which the impedance $\boldsymbol{Z}$ was computed.

\section{Methods and subjects}

ICG and ECG signal registrations. ICG recordings were made using a four-electrode system attached to the left side of the body (figure 1) [5]. The outer two electrodes applied a small alternating $(64 \mathrm{kHz})$ electrical current $i$ of $0.3 \mathrm{~mA}$ (r.m.s.) through the upper part of the body. The inner two electrodes continuously measured the subsequent electrical voltage difference $V(t)$ over the region of the heart, from 
which the electrical impedance $\boldsymbol{Z}(\boldsymbol{t})$ was computed continuously. The measuring electrodes were placed on the left side of the body just below the clavicle and at the level of the xiphoid process. The current supplying electrodes were placed on the forehead and at least $20 \mathrm{~cm}$ below the lower measuring electrode, at the level of the anterior superior iliac spine, both at an adequate distance of the measuring electrodes to avoid voltage measurement in the inhomogeneous regions of the electrical field in the vicinity of the current supplying electrodes [8]. The ECG signal was derived simultaneously from the two inner electrodes.

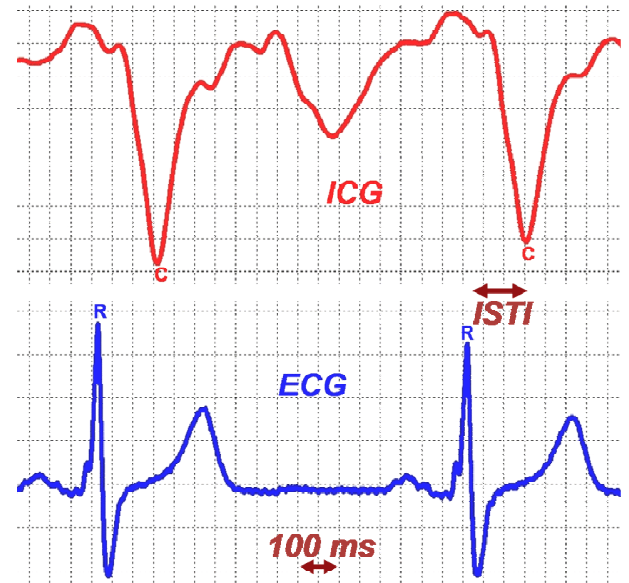

Fig.2: Simultaneous registration of an Impedance CardioGram (ICG) and an ElectroCardioGram (ECG) (arbitrary units). ISTI is defined as the time interval between the R-point in the ECG and the C-point in the ICG.

Figure 2 shows an example of a simultaneous recording of an ICG and an ECG signal in the same subject. The ICGsignal is the first time-derivative of the impedance variations, $d Z / d t$, across the heart. ISTI is defined as the time interval between the R-point in the ECG and the Cpoint in the ICG.

Recordings were made using an impedance cardiograph custom designed and built at the Department of Physics and Medical Technology, VU University Medical Center, Amsterdam, the Netherlands. The signals were ADconverted using an ADInstruments PowerLab 8SP and processed on a personal computer using ADInstruments Chart (version 5.1.1) for Windows.

Subjects and procedures. Two groups of subjects were tested: patients with PD and age-matched healthy subjects. All patients fulfilled the clinical diagnostic criteria of the United Kingdom Parkinson's Disease Society Brain Bank, and were selected from the outpatient clinic for movement disorders of the Neurology department of the VU University medical center. Inclusion was limited to patients in Hoehn and Yahr stages 1-3. The sample consisted of 11 male and 8 female patients, having an age (mean \pm s.d.) of $61 \pm 7$ years (range: $50-73 \mathrm{yrs}$ ), height of $175 \pm 10 \mathrm{~cm}$ (range: $154-188 \mathrm{~cm}$ ) and body mass of $77 \pm 15 \mathrm{~kg}$ (range: $52-117 \mathrm{~kg}$ ). The control group of healthy subjects was mainly recruited among the partners of the PD-patients and the staff of the medical centre. This group consisted of 13 males and 11 females, with an age (mean \pm s.d.) of $61 \pm 7$ years (range: $51-74 \mathrm{yrs}$ ), a height of $173 \pm 8 \mathrm{~cm}$ (range: $155-186 \mathrm{~cm}$ ) and a body mass of $77 \pm 16 \mathrm{~kg}$ (range: 55 $109 \mathrm{~kg}$ ). Subjects and patients using beta-blockers or antiarrhythmic agents were excluded from the study because of the influence of these drugs on heart activity. Recordings were made in a supine resting state during one minute of normal breathing. Subsequently the patients and subjects were requested to breath deeply at a rate of $0.1 \mathrm{~Hz}$ during one minute, also in a supine position. Vocal instruction was used to indicate the periods of five seconds in- and five seconds exhalation. The variability in the RR-intervals and the ISTI's was calculated from the maximal, minimal and mean interval length $(\mathrm{IV})$ during this minute according to the method of Shahani et al [9]:

$$
V A R=100 * \frac{I V_{\max }-I V_{\min }}{I V_{\text {mean }}}
$$

This calculation resulted in the outcome measures RR-VAR and ISTI-VAR in the two groups of subjects, during normal breathing at rest and during stimulated breathing. Significance of differences within the groups was tested using the paired Wilcoxon signed rank test and between the two groups using the unpaired Mann-Whitney test. A level of $p<0.05$ was considered to be significant. The study was approved by the local medical ethics committee and all subjects gave their written informed consent.

\section{Results}

No differences were found both in RR-interval between the two groups, or within the groups between the two conditions: normal breathing at rest and deep, stimulated breathing at $0.1 \mathrm{~Hz}$. The results are listed in the table 1 .

Table 1. RR-interval \pm S.D. $[\mathrm{ms}]$ in the group of patients with Parkinson's disease and in the group of control subjects while breathing at rest and during stimulated, deep breathing at $0.1 \mathrm{~Hz}$.

\begin{tabular}{lll}
\hline subjects & $\begin{array}{l}\text { RR }[\mathrm{ms}] \\
\text { at rest }\end{array}$ & $\begin{array}{l}\text { RR }[\mathrm{ms}] \\
\text { stimulated }\end{array}$ \\
\hline controls (24) & $990 \pm 180$ & $990 \pm 170$ \\
patients (19) & $940 \pm 170$ & $940 \pm 170$ \\
\hline
\end{tabular}

No differences were found also in ISTI-interval between the two groups, or within the groups between the two conditions. The results are listed in the table 2 .

Table 2. ISTI-interval \pm S.D. [ms] in the group of patients with Parkinson's disease and in the group of control subjects while breathing at rest and during stimulated, deep breathing at $0.1 \mathrm{~Hz}$.

\begin{tabular}{lll}
\hline subjects & $\begin{array}{l}\text { ISTI }[\mathrm{ms}] \\
\text { at rest }\end{array}$ & $\begin{array}{l}\text { ISTI [ms] } \\
\text { stimulated }\end{array}$ \\
\hline controls (24) & $145 \pm 14$ & $147 \pm 10$ \\
patients (18) & $143 \pm 17$ & $147 \pm 19$ \\
\hline
\end{tabular}


The variability, however, of the RR-interval, as indicated by RR-VAR, was higher during deep metronomic breathing than in the resting condition for both the control group $(\mathrm{p}<0.01)$ and the patient group $(\mathrm{p}<0.025)$. Although the RR-VAR increased during deep breathing in both groups, the RR-VAR was significantly higher in the control group than in the PD group $(\mathrm{p}<0.025)$. There was no significant difference in RR-VAR between both groups breathing at rest $(\mathrm{p}>0.05)$. The results are listed in table 3 .

Table 3. Variability in RR-interval (RR-VAR) \pm S.D. [\%] in the group of patients with Parkinson's disease and in the group of control subjects while breathing at rest and during stimulated, deep breathing at $0.1 \mathrm{~Hz}$.

\begin{tabular}{lll}
\hline subjects & $\begin{array}{l}\text { RR-VAR [\%] } \\
\text { at rest }\end{array}$ & $\begin{array}{l}\text { RR-VAR [\%] } \\
\text { stimulated }\end{array}$ \\
\hline controls (24) & $19 \pm 10$ & $31 \pm 12$ \\
patients (19) & $17 \pm 7$ & $23 \pm 10$ \\
\hline
\end{tabular}

RR-VAR in both groups and in both conditions are presented graphically in figure 3 .

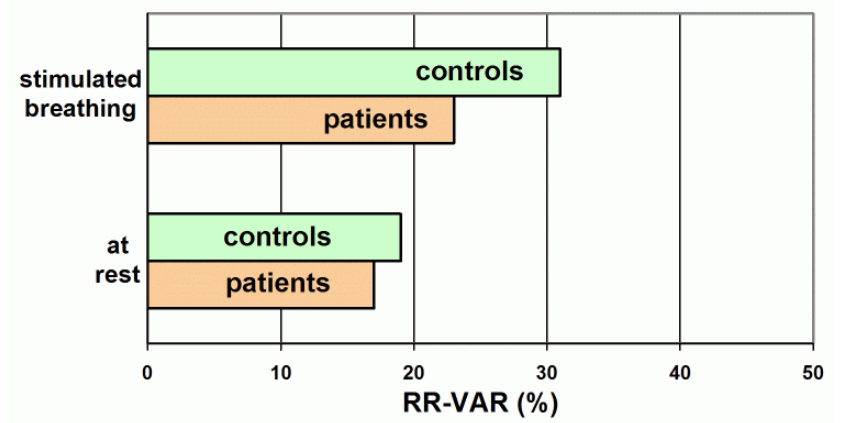

Fig.3: Variability in RR-interval (RR-VAR) in the group of patients suffering from Parkinson's disease and in the control group, during normal breathing at rest and during stimulated, deep breathing. At rest there is no significant difference between the two groups. During stimulated deep breathing RR-VAR increased significantly within both groups. However, this increase was significantly less in the patient group.

The variability in ISTI at rest, as indicated by ISTI-VAR, was higher in patients than in control subjects $(p<0,05)$. The variability increased significantly during deep breathing in the control group $(\mathrm{p}<0.01)$ but did not change significantly in the patients group. The results are listed in table 4 .

Table 4. Variability in ISTI (ISTI-VAR) \pm S.D. [\%] in the group of patients with Parkinson's disease and in the group of control subjects while breathing at rest and during stimulated, deep breathing at $0.1 \mathrm{~Hz}$.

\begin{tabular}{lll}
\hline subjects & $\begin{array}{l}\text { ISTI-VAR [\%] } \\
\text { at rest }\end{array}$ & $\begin{array}{l}\text { ISTI-VAR [\%] } \\
\text { stimulated }\end{array}$ \\
\hline controls (24) & $23 \pm 15$ & $34 \pm 17$ \\
patients (18) & $44 \pm 40$ & $38 \pm 20$ \\
\hline
\end{tabular}

ISTI-VAR in both groups and in both conditions are presented graphically in figure 4 .

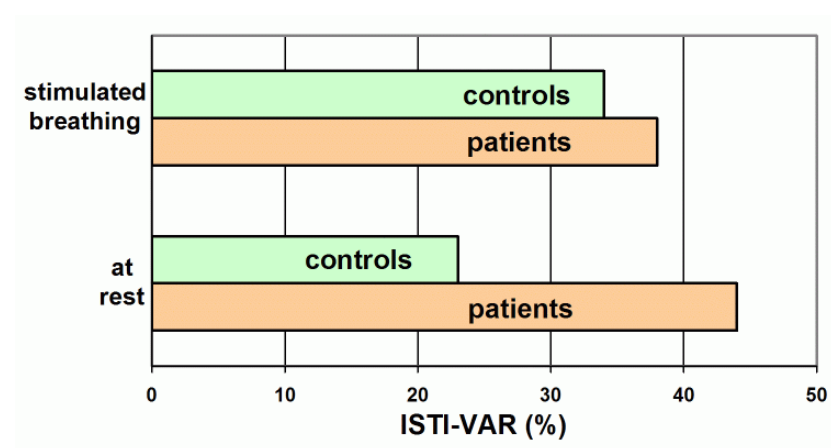

Fig.4: Variability in ISTI (ISTI-VAR) in the group of patients suffering from Parkinson's disease and in the control group, during normal breathing at rest and during stimulated, deep breathing. At rest there is a significant difference between the two groups. During stimulated deep breathing ISTI-VAR increased significantly in the control group. However, ISTIVAR did not change significantly in the patient group as it was already substantially elevated.

\section{Discussion}

The observations in patients with PD demonstrate that measurement of ISTI can be useful as an addition to cardiovascular reflex testing in diagnosing autonomic dysfunction. No significant differences were found in RR and ISTI between the PD patient group and the control group at rest or during stimulated, deep breathing. The variability, however, of RR and ISTI, as measured by RRVAR and ISTI-VAR, showed substantial differences between PD patients and controls. The patients showed a smaller increase in RR-VAR than the controls during stimulated deep breathing. These findings are consistent with reports that variability in RR-interval, caused by breathing, is dominated by the parasympathetic nervous system, which is affected by Parkinson's disease[10,11]. In healthy circumstances, the cyclic changes in intra-thoracic pressure are registered by baro-receptors inside the thorax. The parasympathetic system continuously adjusts to these changes in pressure, resulting in an increased $R R$ variability. In the PD patients, the RR variability during stimulated deep breathing was significantly lower than in the controls. This observation reflects reduced parasympathetic activity in response to changes in intrathoracic pressure in the patient group and confirms the results of previous studies on RR variability [10,11].

ISTI variability was significantly higher in PD patients than in controls during normal breathing at rest. It increased during deep metronomic breathing in the control group, but not in the patient group as it was already high during normal breathing. ISTI is directly related to the pre-ejection period (PEP) [5], which is an index of contractility and only influenced by sympathetic but not by parasympathetic activity in humans $[12,13,14]$. Apparently the data show that the variability in sympathetic activity at rest was higher in patients than in controls. A possible explanation is that, in PD patients, the sympathetic system has to compensate for the decreased response of the parasympathetic system to 
level blood pressure variations caused by breathing. During stimulated deep breathing, the ISTI variability increased significantly in controls but not in PD patients. This result can be explained by assuming that the high ISTI variability in PD patients was already at a maximal level at rest. This limited compensatory mechanism of the sympathetic system also explains the reduced RR variability in PD patients during deep breathing. This mechanism appears to be at a maximal level in normal breathing and fails in compensating during deeper breathing. As a consequence, the RR variability is limited in PD patients in comparison to controls.

It is not likely that these observations simply resulted from a normal difference in the mechanics of the ventilation between the two groups $[15,16]$. If the lower RR variability in PD patients during stimulated breathing were the result of a poor breathing performance, the ISTI variability would have been lower as well. If the higher ISTI variability in PD patients in rest were the consequence of deeper breathing in rest, the RR variability in rest would have been higher as well. Neither was observed.

\section{Conclusions}

From these results it is concluded that ISTI is a practical, additional and independent parameter that can be used to assist other tests in evaluating autonomic control of the heart in Parkinson's disease. The necessary technical equipment is relatively inexpensive and simple to operate. The measurement of ISTI is non-invasive and can be used for monitoring purposes. The measurement, however, is not limited to a clinical environment or to clinical applications.

\section{Acknowledgement}

This study was financially supported by the Alkemade Keuls Foundation, project \#VI-03.

\section{References}

1. Visser KR, Lamberts R, Korsten HHM, Zijlstra WG. Observations on blood flow related electrical impedance changes in rigid tubes. Pflügers Arch. 1976; 366: 289-91.

2. Sakamoto K, Muto K, Kanai H, Lizuka M. Problems of impedance cardiography. Med \& Biol Eng \& Comput. 1979; 17: 697-709
3. Patterson, RP. Impedance cardiography : What is the source of the signal? J Phys: Conf Ser. 2010; 224012118 http://dx.doi.org/10.1088/1742-6596/224/1/012118

4. Bernstein DP. Impedance Cardiography: Pulsatile blood flow and the biophysical and electrodynamic basis for the stroke volume equations. J Electr Bioimp. 2010; 1: 2-27.

5. Meijer JH, Boesveldt S, Elbertse E, Berendse HW. Method to measure autonomic control of cardiac function using time interval parameters from impedance cardiography. Physiol Meas. 2008; 29 S383-91.

6. Lababidi Z, Ehmke DA, Durnin RE, Leaverton PE, Lauer RM. The first derivative thoracic impedance cardiogram. Circulation. 1970; 41: 651-8.

7. Gerritsen J, TenVoorde BJ, Dekker JM, Kingma R, Kostense PJ, Bouter LM, and Heethaar RM. Measures of cardiovascular autonomic nervous function: agreement, reproducibility, and reference values in middle age and elderly subjects. Diabetologia. 2003; 46: 330-38.

8. Meijer JH, Reulen JPH, Oe PL, Allon W, Thijs LG, Schneider H. Differential impedance plethysmography for measuring thoracic impedances. Med \& Biol Eng. \& Comput. 1982; 20: 187-94.

9. Shahani BT, Day TJ, Cros D, Khalil N, Kneebone CS. RR interval variation and the sympathetic skin response in the assessment of autonomic function in peripheral neuropathy. Arch Neurol. 1990; 47: 659-64.

10. GurevichT Y, Groozman GB, Giladi N, Drory VE, Hausdorff $\mathrm{JM}$, Korczyn AD. R-R interval variation in Parkinson's disease and multiple system atrophy. Acta Neurol Scand. 2004; 109: 276-9.

11. Zakrzewska-Pniewska B, Jamrozik Z. Are electrophysiological autonomic tests useful in the assessment of dysautonomia in Parkinson's disease? Parkinsonism Relat Disord. 2003; 9: 179-83.

12. Newlin DB, Levenson RW. Pre-ejection period: measuring beta-adrenergic influences upon the heart. Psychophysiology. 1979; 16: 546-53.

13. Willemsen GH, de Geus, EJ, Klaver CH, van Doornen LJ, Carroll D. Ambulatory monitoring of the impedance cardiogram. Psychophysiology. 1996; 33: 184-93.

14. Schächinger H, Weinbacher M, Kiss A, Ritz R, Langewitz W. Cardiovascular indices of peripheral and central sympathetic activation. Psychosomatic Med. 2001; 63: 788-96.

15. Verceuil L, Linard JP, Wuyam B, Pollak P, Benchetrit G. Breathing pattern in patients with Parkinson's disease. Resp Physiol. 1999; 118: 163-72.

16. Shaheen HA, Ali MA, Abd Elzaher MA. Parkinson's disease and pulmonary dysfunction. Egypt $\mathrm{J}$ Neurol Psychiat Neurosurg. 2009; 46: 129-40. 